\title{
Oculo-auriculo-vertebral spectrum with myopathy and velopharyngeal insufficiency. A case report with a non-branchiomeric muscle biopsy
}

\author{
Giovanni Murialdo,1 Attilia Piazzi,2 Giuseppe Badolati,2 Enrico Calcagno,2 Agostino Berio² \\ 1 Department of Internal Medicine and Medical Specialties, University of Genoa; ${ }^{2}$ Department of \\ Neurosciences, Rehabilitation, Ophthalmology, Genetics and Mother-Child Sciences, University of \\ Genoa, Genoa, Italy
}

\begin{abstract}
In the present paper we report on a case of oculo-auriculo-vertebral spectrum presenting fluorescence in situ hybridization and comparative genomic hybridization tests negative, hypotonia of some branchiomeric muscles (with velo-pharyngeal insufficiency, dysphagia and nasal voice) and non-branchiomeric muscles (with strabismus and limb hypotrophy). On the basis of the left quadriceps muscle biopsy, showing anisometry and prevalence of type 1 fibers, and on literature data, we underline the relevance of $T B X 1$ gene (regulator of neural crest cells and activator of myogenic factors in branchiomeric muscles development) and of PAX3 gene (present in neural crest, inducing migration of these cells and reported in non-branchiomeric muscles). We conclude that the case of OAVS presented a generalized myopathy and we hypothesize that a cluster of genes strictly neural crest cells related, including $T B X 1$ and $P A X 3$, may be responsible of the branchiomeric and non-branchiomeric myopathy; alternatively, a regulatory mechanism abnormally common to OAVS and velo-cardio-facial syndrome could be present.
\end{abstract}

\section{Introduction}

In oculo-auriculo-vertebral spectrum (OAVS), ${ }^{1}$ hypotonia, muscular hypotrophy, and hypernasal voice were repeteadly reported.1,2 In the

Correspondence: Agostino Berio, Department of Neurosciences, Rehabilitation, Ophthalmology, Genetics and Mother-Child Sciences, University of Genoa, largo G. Gaslini 5, 16121 Genoa, Italy.

Tel. +39.010 .56362248 - Fax: +39.010 .3538265 .

E-mail: agostinoberio@ospedale-gaslini.ge.it

Key words: Oculo-auriculo-vertebral spectrum; Non-branchiomeric muscle biopsy; Neural crest cells; Velopharyngeal insufficiency.

Acknowledgements: the authors thank Anna Capurro for her help in revising the manuscript.

Received for publication: 17 July 2015.

Accepted for publication: 27 November 2015.

This work is licensed under a Creative Commons Attribution NonCommercial 4.0 License (CC BY-NC 4.0).

(c) Copyright G. Murialdo et al., 2016

Licensee PAGEPress, Italy

La Pediatria Medica e Chirurgica 2016; $38: 121$

doi:10.4081/pmc.2016.121 personal case of OAVS with these symptoms reported below, the a nonbranchiomeric muscle (left quadriceps) biopsy showed generalized muscle disease. This case emphasizes the importance and complexity of the myopathy in OAVS.

\section{Case Report}

Family history of the proposita showed a grandmother with right eye proptosis and microphthalmia, the mother with mild hearing loss, a brother with right hemifacial microsomia and right eye smaller than left and a nephew with congenital talipes varus.

The proposita was born by caesarean section, birth weight was 3150 $\mathrm{g}$ and head circumference was $38 \mathrm{~cm}$. The child presented with bilateral talipes valgus, right palpebral ptosis, feeding difficulties during the first months of life, delayed developmental milestones and frequent otitis.

At 1 year 7 months, physical examination showed a child in the $75^{\text {th }}$ percentile for height and in the 90 th percentile for weight (Italian growth charts). She presented frontal bossing, right eye microphthalmia with eyelid ptosis and strabismus, normal eye movements, antimongoloid palpebral slant, asymmetric face (right<left), malar hypoplasia, lower right ear with hypoplastic lobules, receding chin, flat nasal root and downturned pyramid, irregular teeth with enamel dysplasia. Dysphagia was present. The voice was hypernasal; during speech the velum palatinum moved but a mild fissure was appreciated between the velum and posterior pharyngeal walls, mainly laterally. During rhinopharyngoscopy, air bubbles were seen in blowing, because pharyngeal constrictor muscles were unable to come into contact with the posterior palatinum velum, with insufficient closure of the gate of pharynx (velo-pharyngeal insufficiency). Hearing was diminished on the right side for the high frequency sound.

The patient showed marked hypotrophy and hypotonia of limb muscles, bilateral talipes valgus, brachyphalangy of thumbs, dorsal lardosis and dorso-lumbar scoliosis. Mild ataxia in walking was present. Radiological examination showed: bone age 2 years 6 months, frontal bossing, right and left temporo-mandibular fossae, zygomatic arches asymmetry, right low set hypoplastic maxilla, and retrognathic mandible. Brain computed tomography (CT) and magnetic resonance imaging (MRI) showed mild cerebral atrophy and hydrocephalus; spine radiology demonstrated L5 spondylolisthesis. Oculo-auriculovertebral spectrum was diagnosed. At 4 years of age, the patient presented with precocious puberty.

Laboratory tests showed intermittent hyperlactacidemia (20-33.7 $\mathrm{mg} / \mathrm{dL})(\mathrm{nv}<20)$, low pyruvicemia $(0.24-0.82 \mathrm{mg} / \mathrm{dL})(\mathrm{nv}<0.5)$ and elevated $\mathrm{L} / \mathrm{P}$ ratio. After physical exercise and after a glycidic meal with fructose, lactacidemia reached a higher value $(28-33 \mathrm{mg} / \mathrm{dL})$ followed 
by slow decrease. Lactate dehydrogenase (LDH) was intermittently high (294-519 IU/dL) (nv 84-361). Fluorescence in situ hybridization (FISH) and comparative genomic hybridization (CGH) tests were negative. Electromyography showed some motor unit potentials, but with reduced duration; motor was amplitude normal.

At somatosensory evoked potentials (lower limbs), cortical components of somatosensory conduction were not identifiable; peripheral components were normal.

Muscular biopsy (left quadriceps m.) (at 13 years of age) showed fibre anisomery with normal, hypotrophic and numerous hypertrophic fibres. No degeneration signs were present, there was no pathologic accumulation of glycides nor of lipids on oil ed and Periodic acid-Schiff staining. On Gomori trichrome stain, no ragged red fibres were recorded; nicotinamide adenine dinucleotide, succinate dehydrogenase, cytochrome c oxidase, phosphorylase, phosphofructokinase, ATP-ase stains was normal. On ATP-ase stain there was a clear prevalence of first type fibres (oxidative fibres) (in normal children, from 30 months to adult age, an equal proportion of type 1 and type 2 fibres was reported by Dubowitz). ${ }^{3}$

To sum up, signs of abnormality in muscles of facial expression, of deglutition and phonation with nasal speech and of limbs were present in our case of OAVS. The gaps in velopharingeal sphincter were probably due both to velar and pharyngeal muscles, necessary for velo-pharyngeal closure, and to hypotonia and hypoplasia.4 This condition may be consequent to OAVS malformative complex involving the facial structures and also other parts of the body. ${ }^{2}$ Palpebral ptosis due to hyposthenia of levator palpebrae muscle, pharynx constrictor and levator velipalatini muscles, and limb muscle hypotonia and hypotrophia were clinical signs of myopathy, confirmed by hyperlactacidemia and elevated $\mathrm{LDH}_{2}^{2}$ We may conclude that, in our patient, myopathy affected branchiomeric and nonbranchiomeric muscles (quadriceps m.) (Table 1).

\section{Discussion}

Oculo-auriculo-vertebral spectrum is characterized by cardinal features of hemifacial microsomia, eye, ear, face and vertebral abnormalities, ${ }^{1}$ sometime associated with multiple malformations. ${ }^{1}$ Goldenhar syndrome, a typical neurocristopathy, ${ }^{5-6}$ is included in OAVS.

The OAVS syndrome present multiple etiologies. It was frequently observed in children born from diabetic mothers. ${ }^{7}$ In some cases, OAVS affected individuals in subsequent generations with dominant autosomal or recessive inheritance. ${ }^{8-10}$ In these families, as in that of our patient, some members may present with isolated features. The disease occurs in monozygotic twins. These observations confirm that genetic factors can increase susceptibility to OAVS.11 The estimate risk of recurrence of OAVS in these families is $1-2 \% .1$ The disease is considered a neurocristopathy.5,6-10 The alterations of facial osseus, cartilagineous, vascular and peripheral nerve structures are attributed to aberrant migration to the first and second branchial arches during $4^{\text {th }}$ and $5^{\text {th }}$ embryonic weeks and/or integration with tissues of neural crest cells (NCC). ${ }^{10}$ In NCC, specific expression of some homeobox genes (MSX, PAX3) $)^{11,12}$ and some factors (FGF8, FGF10) are important for the differentiation of ectodermal and mesenchymal tissues in the first and second branchial arches, which leads to various important structures. In pathological conditions, an abnormality of NCC leads to alterations of the developing ear and eye (during the $4^{\text {th }}, 5^{\text {th }}$ and $6^{\text {th }}$ week)..$^{10-13}$

In OAVS, the clinical variability is high also in familial cases, but no specific gene for OAVS was demonstrated and the disease was attributed to a contiguous gene dysfunction. ${ }^{14}$ Epigenetic factors as disruption of $B A X$ were also advocated.

The generalized malformations present in this condition suggest a disorder of blastogenesis during the first 28 days of embryological life, when body axes are formed and cardio-angiogenesis, meso-nephrogenesis, neurulation and somites arise. ${ }^{10}$ Neurulation with migration of NCC is essential for the development of branchial arches, from which face and neck structures derive.

In humans, five pharyngeal arches (i.e. a series of transitory cartilagineous structures surrounding the pharynx) arise in the lateral side of the head during the $4^{\text {th }}$ and $5^{\text {th }}$ weeks of embryological life. Each arch presents endodermal, ectodermal and mesodermal cells, and NCCderived cells. The endoderm originates the limiting epithelium, the thyroid, the parathyroids, and the thymus; the ectoderm originates the epidermal structure; the paraxial and anterior occipital mesoderms originate the mesodermal core of every arch which is populated by NCC contributing to mesenchime which in turn gives rise to connective, cartilagineous, and bone tissues of the head, face, and neck.,5,-10 The mesodermal cells of pharyngeal arches migrate laterally in the pharyngeal region, giving rise to the branchiomeric skeletal muscles of the head and neck, ${ }^{15-17}$ i.e. the muscles of mastication, deriving from the $1^{\text {st }}$ arch, the muscles of facial expression, deriving from the $2^{\text {nd }}$ arch, the muscle of the pharynx and the intrinsic muscle of the larynx, originating from the $3^{\text {rd }}$ and $4^{\text {th }}-5^{\text {th }}$ arches (Table 1)..$^{15,16}$

The non-branchiomeric muscles of the head include that muscles derived from the most anterior part of the paraxial mesoderm: extraocular muscles (medialis rectus, inferior rectus, inferior obliquus, levator palpebrae), tongue muscles originating in the anterior somites, and trunk and limb muscles, arising from somites.

Somites are spheres of epithelial cells adjacent to the neural tube and notochord; they become mesenchymal in the ventral part (sclerotome) from which the vertebrae arise, and persist in the dorsal part (dermomyotome), giving rise to skeletal muscles and dermis. The $S h h$ gene, which stimulates somatic myogenesis in mouse, was demonstrated in floor plate notochord and in pharyngeal arches, ${ }^{13}$ Wnt- 1 and Wnt- 3 arise in

Table 1. Branchiomeric and non-branchiomeric muscles of head and neck.

$$
\text { Branchiomeric muscles of head and neck }
$$

\section{Muscle of mastication}

Masseter

Pterygoid

Temporalis

Anterior belly of digastric

Tensor palatini

Tensor tympani

\begin{tabular}{ll} 
Muscles of facial expression & Buccinator \\
& Auricularis \\
& Posterior belly of digastric \\
& Stylo(jo)ideus \\
& Frontalis \\
& Orbicularis oculi \\
& Orbicularis oris \\
\hline Muscles of pharynx & Stylopharyngeous \\
& Pharynx constrictor \\
& Levator veli palatini \\
\hline Muscles of larynx & \\
\hline Non-branchiomeric muscles of head
\end{tabular}

Extraocular muscles

Rectus medialis

Rectus inferior

Obliquus inferior

Levator palpebrae

Tongue muscles

Trunk muscles

Limb muscles 
the neural tube and induce in association to $S h h$, trunk somatic myogenesis. ${ }^{18}$ During embryological life, the muscle which first differentiates is the myotome, originated by signals of adjacent tissues (neural tube, notochord). The myogenic progenitor cells of the dermomyotome give rise to all non-branchiomeric skeletal muscles of the body (trunk and limb) ${ }^{18}$ and to some head muscles. In the dermomyotome, some genes are expressed: dorsally, $P a x 7$ and Pax3 pathways, which activate myogenic basic-helix loop helix regulatory factors (Basic HLHMRF), Myf5, MyoD and myogenin; ventrally, in sclerotome, Paxl and Pax9.18 Pax3 is required in embryonic skeletal myogenesis ${ }^{19,20}$ and in the developmental program regulating NCC. Pax3 is expressed in paraxial mesodermal18-20 and neuroectodermal tissues, in epithelial somites, ${ }^{21}$ and in mesoangioblasts, vessel-associated stem cells which may differentiate in skeletal muscles and other mesodermal tissues (smooth and cardiac muscles, bones, fat). Pax3 is found in dermomyotome (dorsal somite cells from which skeletal muscles derive) and is required for the migration of these cells outside the somites; subsequently, $\operatorname{Pax} 3$ activates the Myf5 gene in limbs and MyoD, Fgfr4 and Sprouty inhibitory genes. On Pax3 and Pax7 positive cells, originating from the central dermomyotome, depend the differentiation of mesoangioblasts in skeletal muscles and post-natal myogenesis. ${ }^{20}$ In humans, $P A X 3$ mutation causes the Waardenburg-Klein syndrome, a NCC disease with sensory, hearing, pigmentation and muscular defects. ${ }^{22}$

In embryo, non-branchiomeric skeletal muscles development is regulated by the $M y o D$ family of basic HLHMRF, first expressed in branchial arches. ${ }^{4}$ Mice without Myf5 and Pax3 factors do not develop skeletal muscles of trunk and limbs but present normal head muscles. ${ }^{4}$

Wnt signal stimulates myogenesis of the trunk but blocks that of the head. ${ }^{4}$ Therefore, it is evident that branchiomeric muscle development is genetically regulated in a different manner compared to the nonbranchiomeric muscle. In humans, TBXI (homologous to mouse Tbx1) plays a central role in initiating branchiomeric muscle development, ${ }^{13,22-24}$ directly and indirectly, via the activation of MYF5 and MYOD1 ${ }^{4}$ and the modulation of myogenic signals in surrounding NCC; abnormal development of these muscle might explain the swallowing difficulties present in our case. Tbxl gene is expressed in mice in endoderm and mesoderm pharyngeal arches ${ }^{13}$ during NCC migration. ${ }^{24}$ It plays an important role in mandibular arches development by regulating NCC patterning; 4 it is expressed in adult skeletal muscles and in vertebral column. ${ }^{24}$ In heterozygous $T b x 1+/$ - mice all first arch derived branchiomeric muscles are present including pharyngeal constrictor musculus, but they are absent in homozygous Tbxl -/- mice, ${ }^{4}$ a condition attributed to the low Tbxl level.

In patients with velo-cardio-facial syndrome attributed to $22 \mathrm{q} 11.2$ microdeletion and haploinsufficiency of TBX1, Zim et al.23 demonstrated that velo-pharingeal insufficiency with hypernasal speech and swallowing difficulty were due to hypotonia of velar and pharyngeal branchiomeric muscles, particularly the superior pharyngeal constrictor, necessary for pharyngeal closure. In these patients, TBXI haploinsufficiency with reduced $T B X 1$ levels may lead to pharyngeal hypotonia. ${ }^{4-23}$ At muscular biopsy, superior pharyngeal constrictor muscle presented anisometry and a significantly greater proportion of type 1 (oxidative, with more mitochondria) fibres than in normal subjects. ${ }^{23}$ These observation suggest the relevance of $T B X 1$ in branchiomeric myogenesis. ${ }^{23}$

In our case of OAVS, hypotonia observed at birth of non-branchiomeric rectus medialis musculus (with strabismus), of non-branchiomeric muscles as levator palpebrae (with ptosis), and foot muscles (with talipes valgus), suggest a congenital myopathy of non-branchiomeric muscles, with prevalence of type 1 oxidative fibers, more rich in mitochondria. Nasal speech and swallowing difficulties were attributed to velo-pharyngeal branchiomeric muscle insufficiency.

The association of NCC-derived facial structure abnormalities with branchiomeric muscle abnormalities may suggest a causative role of NCC regulatory genes (as TBX1)4 possibly in association with other genes. ${ }^{4}$

In our OAVS case, the cause of non-branchiomeric muscle hypotonia is unknown.

In patients with velo-cardio-facial syndrome due to TBX1 mutation (del 22q11.2), hypotonia of non-branchiomeric muscles (extraocular and limb muscles) and velopharyngeal hypotonia are present. 4 However, the pathophysiology of this condition has not been completely understood yet. 13 The non branchial arch phenotype in man can be explained by the expression pattern of haploinsufficiency of $T B X 1$ in mesoderm and in sclerotome. 13

It was observed in patients with TBX1 (del 22q11.2) that TBX1 is expressed in sclerotome and in mesoderm, that myogenic regulatory factors (MRFs) expression is normal in non-branchiomeric muscles of limbs. Therefore, hypotonia in these muscles cannot be attributed to isolated $T B X 1$ deficiency, but rather to environment or to a different gene background which can modify the effect of haploinsufficiency of gene with the deletion. 25 In the latter case, other genes may contribute to the myopathy of the non-branchiomeric muscles: i.e. NCC-related genes $(M S X)^{26}$ or genes regulating NCC (PAX3). ${ }^{20,21}$ In our case, the result of the biopsy of a non-branchiomeric muscle, similar to that performed by Zim in a velo-cardio-facial syndrome branchiomeric muscle, ${ }^{23}$ suggests the relevance of the NCC regulatory genes (direct or indirect) or of NCC related genes. Negativity of FISH and CGH for a gene mutation cannot rule out the possible complex insertion of genes or minimal deletion(s), rearrangements and consequent haploinsufficiency of genes (as reported in TBX1 non deleted patients).25 An activating factors to OAVS and velocardiofacial/Di George syndrome was recently hypothesized responsible of these conditions. ${ }^{27}$

\section{Conclusions}

In conclusion, our OAVS case presents a generalized myopathy of branchiomeric and non-branchiomeric muscles. In this neurocristopathy NCC-related genes (as TBX1) or genes in NCC (as PAX3) may have some importance in muscular pathophysiology. Further studies are necessary to understand the cause of this complex disease and the role of NCC.

\section{References}

1. Gorlin RJ, Jue KL, Jacobsen U, Goldschmidt E. Oculo-auriculo-vertebral dysplasia. J Pediat 1963;63:991-9.

2. Shprintzen R, Croft CB, Berkman MD, Rakoff SJ. Velopharyngeal insufficiency in the facio-auriculo-vertebral malformation complex. Cleft Palate J 1980;17:132-7.

3. Dubowitz V. Enzyme histochemistry of skeletal muscle. J Neurol Neurosur Ps 1965;28:516-24.

4. Kelly RG, Jerome-Majewska LA, Papaioannou VE. The del 22qII.2 candidate gene TbxI regulates branchiomeric myogenesis. Hum Mol Genet 2004;13:2829-40.

5. Bolande RP. The neurocristopathies. Hum Pathol 1974;95:404-20.

6. Bolande RP. Neurocristopathy: its growth and development in 20 years. Pediatr Pathol Lab Med 1997;17:1-25.

7. Stromland K, Miller M, Sjogreen C, et al. OAV spectrum. Am J Med Genet 2007;143:1317-25.

8. Stoll C, Viville B, Treisser A, Gasser B. A family with dominant oculo-auriculo-vertebral spectrum. Am J Med Genet 1998;78:345-9.

9. Martelli HJR, Miranda RT, Fernanes CM, et al. Goldenhar syndrome: clinical features with orofacial emphasis. J Oral Appl Sci 2010;18:646-9.

10. Berio A, Mangiante G, Oliaro E, Piazzi A. Oculo-auriculo-vertebral 
spectrum with large cerebral cyst: affinity to holoprosencephaly. Minerva Pediatr 2012;64:549-55.

11. Hartsfield JK. Review of the etiologic heterogenity of the OAVS. Orthod Craniofac Res 2007;10:121-8.

12. Gammill LS, Bronner-Frazer M. Neural crest specification: migrating into genomics. Nat Rev Neurosci 2003;4:795-805.

13. Kobrynsky L, Sullivan KE. Velo-cardio-facial syndrome, Di George syndrome: the chromosome 22qII.2 deletion syndromes. Lancet 2007;370:1443-52.

14. Tasse C, Mayewski F, Bohringer S, et al. A family with autosomal dominant oculo-auriculo-vertebral spectrum. Clin Dysmorphol 2007;16:1-7.

15. Lu JR, Bassel-Duby R, Hawkins A, et al. Control of facial muscle development by MyoR and capsulin. Science 2002;298:2378-81.

16. Cohen M. Malformations of the craniofacial region: evolutionary, embryonic, genetic and clinical perspectives. Am J Med Genet 2002;115:245-68.

17. Goodman FR. Congenital abnormalities of body pattering: embryology revisited. Lancet 2003;362:651-62.

18. Maroto M, Reshef R, Munsterberg AE, et al. Ectopic Pax-3 activates Myo D and Myf-5 expression in embryonic mesoderm and neural tissues. Cell 1997;89:139-48.

19. Lorraine-Teller A. Expression of the PAX 2 gene in human embryo. Am J Med Genet 2001;93:65-88.
20. Messina G, Sirabella D, Monteverde S, et al. Skeletal muscle differentiation of embryonic mesoangioblasts requires PAX 3 activity. Stem Cells 2009;27:157-64.

21. Tajbakhsh S, Rocancourt D, Cossu G, Buckingham M. Redefining the genetic hierarchies controlling skeletal myogenesis: Pax -3 and Myf-5 act upstream of Myo D. Cell 1997;89:127-38.

22. Klein D. Albinism partiel (leucisme) avec surdomutism, blepharophimosis, dysplasie myo-articulaire. Helv Paediatr Acta 1950;51:138.

23. Zim S, Schelper R, Kellman R, et al. Thikness and histologic and histochemical properties of the superior pharyngeal constrictor muscle in velocardio-facial syndrome. Arch Facial Plast S 2003;5:503-10.

24. Chieffo C, Garvey N, Gong W, et al. Isolation and characterization of a gene from the Di George chromosomal region homologous to the mouse Tbx gene. Genomics 1997;43:267-77.

25. Gong W, Gottlieb S, Collins J, et al. Mutation analysis of TBX 1 in non-deleted patients with features of DGS/VCFS or isolated cardiovascular defects. J Med Genet 2001;38:e145.

26. Beck A, Hudgins L, Hoyme E. Autosomal dominant microtia and ocular coloboma. Am J Med Genet 2005;134:359-62.

27. Costa dos Santos PA, De Oliveira SF, Freitas EL, et al. Non overlapping $22 q 11.2$ microdeletions in patients with oculo-auriculo-vertebral spectrum. Am J Med Genet 2013;164:551-3. 